EPJ Web of Conferences 71, 00038 (2014)

DOI: 10.1051/epjconf/20147100038

(C) Owned by the authors, published by EDP Sciences, 2014

\title{
Search for a high mass Higgs boson using the ATLAS detector
}

\author{
Sara Diglio ${ }^{1,2, a}$ \\ ${ }^{1}$ The University of Melbourne \\ ${ }^{2}$ visitor at The University of Bern
}

\begin{abstract}
The ATLAS experiment has discovered a new boson with properties consistent with the Standard Model Higgs. The next stage will be to determine whether this Higgslike particle is fully responsible for the generation of the masses of the other Standard Model particles. There are natural extensions to the Standard Model which are compatible with the current results and predict the existence of an additional neutral Higgs-like resonance in the high mass region. Search for a heavy Higgs boson using the ATLAS detector will be presented in the following.
\end{abstract}

\section{Introduction}

The 2012 discovery of a new boson by the ATLAS [1] and CMS [2] collaborations at the LHC is possibly the last missing ingredient of the Standard Model (SM). Measurements of its properties show consistency with predictions of the SM [3-5]. Nevertheless, these measurements do not conclusively establish it as being the SM Higgs boson. It therefore remains important to extend the SM Higgs boson searches to the full explorable mass range. Furthermore, there are extensions of the SM which are compatible with the current results and which predict the existence of additional neutral Higgs-like resonances in the high-mass region $\left(m_{H}>200 \mathrm{GeV}\right)$.

The simplest example of such a SM extension is represented by a model in which the discovered boson mixes with a heavy electroweak singlet [6-11] in order to complete the unitarization of $W W$ scattering at high energies. This singlet field acquires a non-vanishing vacuum expectation value, giving rise to an additional resonance in the high-mass region. In these scenarios, the event kinematics are assumed to be similar to those in SM Higgs events. Furthermore, the couplings and widths of the Higgs-like particles are predicted to scale with respect to those of the SM Higgs boson. In particular, current measurements of signal strength and universal couplings of the $\sim 126$ state, reinterpreted in the simplest scenarios offered by these benchmark models, tend to disfavour a second heavy Higgslike particle with non-negligible natural width [12].

Additional examples of possible extensions to the SM include Two Higgs Doublet Models (2HDMs) [13-15] where a second doublet is added to the Higgs boson, giving rise to five physical states: three neutral ( $\mathrm{h}, \mathrm{H}$, which are $\mathrm{CP}$-even and $\mathrm{A}$, which is $\mathrm{CP}$-odd) and two charged $\left(\mathrm{H}^{ \pm}\right)$. These models introduce two mixing angles $\alpha$ and $\beta$, which describe the mixing of CP-even and CPodd states, respectively. Also, $\tan \beta$ is the ratio of the vacuum expectation values of the two doublets: $\tan \beta=v_{2} / v_{1}$. These general models can be further classified depending on the fermions coupling

\footnotetext{
ae-mail: sara.diglio@cern.ch
} 
to the Higgs sector. In Type I 2HDM models all quarks couple to just one of the Higgs doublets (this includes the fermio-phobic limit scenario). If up-type quarks couple to one Higgs doublet and down-type quarks couple to the other, we have Type II models: a well known examples is the minimal supersymmetric standard model (MSSM). Other types (III and IV) consider different couplings for leptons. Under specific assumptions on the parameters describing these models, the heaviest neutral CP-even state will have properties close to ones predicted for a SM heavy Higgs. In light of these considerations, searches for a heavy SM and SM-like Higgs can be considered as the starting point for future beyond the standard model Higgs interpretations and further analysis.

In the next sessions, SM results of the most sensitive channels in the high mass region (i.e $\mathrm{H} \rightarrow \mathrm{WW}$ and $\mathrm{H} \rightarrow \mathrm{ZZ}$ ) will be presented as well as narrow width approximation (NWA) and 2HDM results in the $\mathrm{H} \rightarrow \mathrm{WW} \rightarrow \mathrm{e} v \mu \nu$ using data delivered by the ATLAS detector. The results will be presented started from the most recent ones.

\section{Heavy Higgs SM and SM-like searches}

\subsection{Theoretical aspects related to the high mass region}

The lineshape of an unstable particle is usually described in Monte Carlo (MC) generators with a Breit-Wigner (BW) distribution. This approximation has an accuracy of the order $\Gamma / m$, where $\Gamma$ is the width and $m$ is the pole mass of the particle, but it breaks down at high Higgs boson mass due to the very large Higgs boson width: as an example $m_{H} \gtrsim 450 \mathrm{GeV}$ gives $\Gamma_{H} / m_{H} \gtrsim 10 \%$. The problem has been discussed in details in Ref. [16] and a more appropriate approach to describe the Higgs lineshape has been proposed, known as Complex Pole Scheme (CPS). The corresponding complete calculation of the lineshape in the $\mathrm{gg} \rightarrow \mathrm{H} \rightarrow \mathrm{VV}$ process (where $\mathrm{V}$ states for either the $\mathrm{Z}$ or the $\mathrm{W}$ vector boson) with assessment of the theoretical uncertainties is presented in Ref. [11].

Another important effect that becomes very large in the high mass region is the interference between the signal and the gg $\rightarrow \mathrm{VV}$ non resonant background [11, 17-20]. For a Higgs boson with a large width, which corresponds to high mass in the SM case, the production cross section as well as kinematic variables are affected by this interference between signal and non resonant background production. This effect is neglible for a SM Higgs boson with $m_{H}<400 \mathrm{GeV}$, but its impact increases with increasing of the Higgs width. In Ref. [11], the interference effect is addressed with a proposal to compute the theoretical uncertainties due to missing higher order perturbation terms in the current available interference estimation. Both lineshape and interference effect have been taken into account to perform the high mass region analyses which will be presented in the next sections.

\section{$2.2 \mathbf{H} \rightarrow \mathbf{W W} \rightarrow \mathbf{e} v \mu \nu$ searches}

\subsubsection{Standard Model and Narrow Width Approximation searches}

A search for a Higgs boson in the $\mathrm{H} \rightarrow \mathrm{WW} \rightarrow \mathrm{e} v \mu \nu$ channel in the range $260 \mathrm{GeV} \leq m_{H} \leq 1 \mathrm{TeV}$ has been performed by the ATLAS experiment [21]. The analysis uses proton-proton collision data at a centre-of-mass energy of $8 \mathrm{TeV}$, corresponding to an integrated luminosity of $20.7 \mathrm{fb}^{-1}$.

The final state topology consists of two oppositely charged leptons and large missing transverse energy $\left(\mathbf{E}_{\mathrm{T}}^{\text {miss }}\right)$. Higgs boson candidates produced in association with zero, one or two jets are included in the analysis. The dominant background contributions come from continuum $W W, t t$ and $W t$ production, all of which produce two $W$ bosons as the signal. Drell-Yan $\left(Z / \gamma^{*} \rightarrow l l\right)$ events can fake the signal when they are reconstructed with significant missing transverse energy, while $W+$ jets production with a jet mis-reconstructed as a lepton can also lead to the same final state. Other diboson processes such 


\section{ICNFP 2013}
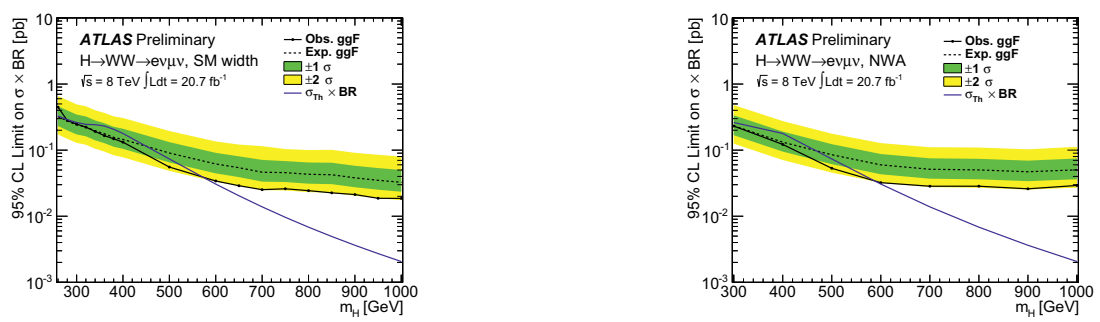

Figure 1. 95\% CL upper limits on the ggF Higgs boson production cross section times branching ratio for $\mathrm{H} \rightarrow$ $\mathrm{WW} \rightarrow l v l v$ (with $l=\mathrm{e}, \mu, \tau$ ) for a scalar with a SM-like (left) and a NWA (right) lineshape [21]. The green and yellow bands show the $\pm 1 \sigma$ and $\pm 2 \sigma$ uncertainties on the expected limit. The SM cross section times branching ratio is shown as a blue line.

as $W \gamma$ and $W Z^{(*)}$ make very small contributions to this high-mass search. The resonance at $m_{H} \sim 126$ $\mathrm{GeV}$ is treated as a background. The requirements on lepton transverse momentum $\left(p_{\mathrm{T}}^{l}\right)$ and dilepton invariant mass $\left(m_{l l}\right)$ have been optimized to minimize this background. Systematic uncertainties arise from both experimental and theoretical sources and differ depending on the jet multiplicity in the final state. The most significant experimental contributions are from the jet energy scale (JES 1-5\%) and resolution (JER $\sim 5-25 \%)$ and the $b$-tagging efficiency ( $\sim 5-12 \%)$. The dominant theoretical contribution belongs to the exclusive jet bin uncertainties on signal $(\sim 38-55 \%)$.

Finally the discriminating variable used in the final likelihood fit to data is the transverse mass $m_{\mathrm{T}}$, defined as $m_{\mathrm{T}}=\left(\left(E_{\mathrm{T}}^{l l}+E_{\mathrm{T}, \text { rel }}^{\text {miss }}\right)^{2}-\left|\mathbf{p}_{\mathrm{T}}^{l l}+\mathbf{E}_{\mathrm{T}}^{\text {miss }}\right|^{2}\right)^{1 / 2}$ with $E_{\mathrm{T}}^{l l}=\left(\left|\mathbf{p}_{\mathrm{T}}^{l l}\right|^{2}+m_{l l}^{2}\right)^{1 / 2}$. The final results are interpreted in the SM scenario as well as in a model-independent context which uses a narrow-width approximation (NWA). A SM Higgs boson within a mass range of $260 \mathrm{GeV} \leq m_{H} \leq 642 \mathrm{GeV}$ is excluded at $95 \% \mathrm{CL}$. To allow for constraints on a new resonance which may have different production rates in the gluon gluon fusion (ggF) and vector boson fusion (VBF) modes, the upper limits are estimated separately for the ggF and VBF production mechanisms. Fig. 1 shows 95\% CL upper limits on the production cross sections times branching ratio (BR) as a function of the Higgs mass, for $\mathrm{ggF}$ in both SM and NWA scenarios. The theoretical SM prediction curve is also superimposed to the limit plot.

\subsubsection{Two Higgs Doublet Model}

The ATLAS collaboration has analyzed the decay chain $\mathrm{H} \rightarrow \mathrm{WW} \rightarrow \mathrm{e} v \mu \nu$, within $2 \mathrm{HDM}$ scenarios, under the hypothesis that the discovered boson at $\sim 126 \mathrm{GeV}$ is the light Higgs (h) and that $\mathrm{A}$ and $\mathrm{H}^{ \pm}$are heavy enough not to affect the cross section and branching ratio of $\mathrm{H}$. The analysis has been performed over the mass range $135 \mathrm{GeV} \leq m_{H} \leq 300 \mathrm{GeV}$ using $13 \mathrm{fb}^{-1}$ of $p p$ collision data at $\sqrt{s}$ $=8 \mathrm{TeV}$ [22]. The main backgrounds and systematics have been assumed to be the same as in the SM case. Final states with either zero or two jets have been studied using a Neural Network approach. The results have been interpreted in the $m_{H}$ vs $\cos \alpha$ plane for a set of values of $\tan \beta$ and limits have been set. 

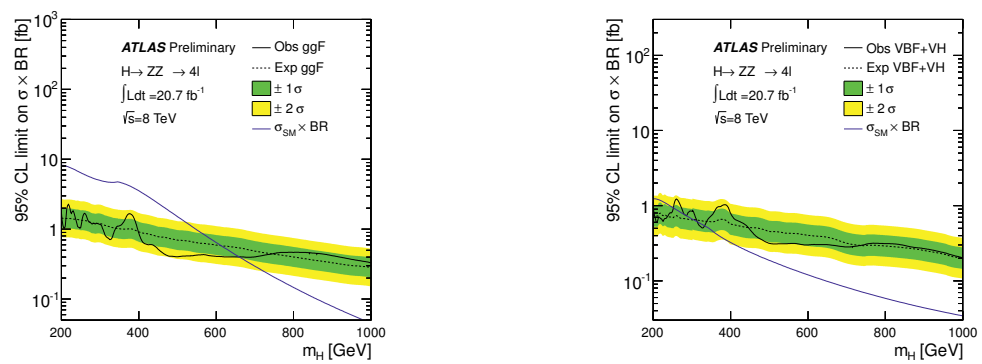

Figure 2. Expected and observed 95\% CL upper limit on the production cross section times branching ratio of $\mathrm{H} \rightarrow \mathrm{ZZ} \rightarrow 4 l$ (where $l=\mathrm{e}, \mu$ ) for a $\mathrm{ggF}$ (left) and VBF-like/VH-like (right) produced SM-like signal as a function of $m_{H}$ [23]. The expected SM cross sections times branching ratio are also presented (blue line).

\section{$2.3 \mathrm{H} \rightarrow \mathrm{ZZ} \rightarrow 4 l$ searches}

The $\mathrm{H} \rightarrow \mathrm{ZZ} \rightarrow 4 l$ (where $l$ can be either a muon or an electron) is the cleanest channel among the high mass sensitive ones because the Higgs boson invariant mass is fully reconstructed. Despite the low BR, its signal to background ratio is higher than the other channels providing a good sensitivity over a wide mass range.

Proton-proton collision data corresponding to an integrated luminosity of $20.7 \mathrm{fb}^{-1}$ collected at $8 \mathrm{TeV}$ have been analysed up to $m_{H}=1 \mathrm{TeV}$ [23]. The largest background in this search comes from continuum $\left(Z / \gamma^{*}\right)\left(Z / \gamma^{*}\right)$ irreducible background and it is estimated using MC simultation normalized to the theoretical cross section. The rate and the composition of the reducible $l l+$ jets and $t t$ backgrounds are evaluated with data-driven methods. For each possible combination of the four leptons in the final state, three different categories are considered: the vector boson fusion (VBF-like), the associate production (VH-like), the gluon gluon fusion (ggF-like). In order to distinguish among categories, firstly the VBF-like one is selected by requiring two high $p_{T}$ jets with large rapidity gap in the invariant mass of the di-jet system $\left(m_{j j}\right)$, events which satisfy such criteria and have an additional electron or muon in the final state are classified as VH-like, all the other events which do not belong neither to VBF-like nor to $\mathrm{VH}$-like categories, are classified as ggF-like. One of the main contribution to the uncertainty belongs to the limited statistics in the high mass region. The results from the event categorisation are used to extract upper limits on production cross sections. The upper limits are estimated separately for the ggF-like and the combined VBF-like/VH-like production mechanisms, as in Fig. 2.

\section{$2.4 \quad \mathbf{H} \rightarrow \mathbf{W W} \rightarrow l v j j$ searches}

Proton proton collision data corresponding to $4.7 \mathrm{fb}^{-1}$ recorded at a centre-of-mass energy of $7 \mathrm{TeV}$ have been used to perform a search for the SM Higgs boson in the $\mathrm{H} \rightarrow \mathrm{WW} \rightarrow l v j j$ channel (where $l=\mathrm{e}$ or $\mu$ ) in the $m_{H}$ range between 300 and $600 \mathrm{GeV}$ [24]. In order to maximize the acceptance for both ggF and VBF production processes, the analysis includes three different categories according to the number of jets (zero, one or two) produced in association to the Higgs boson candidates. In all jets categories the main background comes from the $\mathrm{W}+$ jets production. A combination of $\mathrm{MC}$ and data-driven methods is used to understand the background composition. The background modelling is validated in $m_{j j}$ sidebands with $m_{j j}$ just below $\left(45 \mathrm{GeV}<m_{j j}<60 \mathrm{GeV}\right.$ ) or just above (100 $\mathrm{GeV}<m_{j j}<115 \mathrm{GeV}$ ) the $\mathrm{W}$ boson peak. Similarly to the $\mathrm{H} \rightarrow \mathrm{WW} \rightarrow \mathrm{e} v \mu \nu$ case, the dominant 
experimental systematic uncertainties come from the JES, JER and $b$ tagging veto efficiency. The main theoretical systematic uncertainty is instead related to the lineshape and interference effects discussed in section 2.1. It has been estimated using the empirical formula: $150 \% \times m_{H}^{3}$ (where $m_{H}$ is expressed in $\mathrm{TeV}$ ).

The signal is extracted by fitting the $l v j j$ invariant mass $m_{l v j j}$ reconstructed using the $l v$ invariant mass constraint $m_{l v}=m_{W}$ and the requirement that two of the jets in the event must be consistent with a W $\rightarrow j j$ decay. For zero or one jet cases, the best sensitivity has been reached for $m_{H}=400 \mathrm{GeV}$, where the observed (expected) 95\% confidence level upper bound on the cross section is $2.2 \mathrm{pb}$ (1.9 pb), corresponding to 1.9 (1.6) times the SM prediction. In the two jets category, which is more sensitive to the VBF process, the observed (expected) $95 \%$ confidence level upper bound on the cross section for $m_{H}=400 \mathrm{GeV}$ is $0.7 \mathrm{pb}(0.6 \mathrm{pb})$, corresponding to 7.9 (6.5) times the SM prediction.

\section{$2.5 \mathrm{H} \rightarrow \mathrm{ZZ} \rightarrow l l j j$ searches}

A search for a SM Higgs boson decaying via $\mathrm{H} \rightarrow \mathrm{ZZ} \rightarrow l l j j$ (where $l$ can be either an electron or a muon) has been performed over the mass range $200 \mathrm{GeV} \leq m_{H} \leq 600 \mathrm{GeV}$ [25]. It is based on proton proton collision data recorded at a centre-of-mass energy of $7 \mathrm{TeV}$ and corresponding to an integrated luminosity of $4.7 \mathrm{fb}^{-1}$. In order to optimise the expected sensitivity a distinction between events containing at least two or less than two $b$ tagged jets has been applied as well as a different selection criteria for events with $m_{H}<300 \mathrm{GeV}$ or $m_{H} \geq 300 \mathrm{GeV}$. The main background to this analysis comes from the $\mathrm{Z}$ boson production in association with jets. The shapes of the relevant kinematic distributions for this background are taken from MC simulation, with a small data-driven correction for the low- $m_{H}$ less than two b-tagged jets selection, while the normalisations for all selections are derived directly from data. The flavor composition of the $\mathrm{Z}+$ jets events is derived from $\mathrm{MC}$ and the overall $\mathrm{Z}$ jets normalisation is obtained by fitting the $m_{l l j j}$ distribution in $m_{j j}$ sidebands. The main systematic uncertainties in this channel are the same as discussed in the previous session: JES, JER, b-tagging efficiency as experimental contributions, lineshape and interference effects from the theory. The signal is extracted by fitting the $l l j j$ invariant mass $m_{l l j j}$. Finally the upper limits at $95 \% \mathrm{CL}$ on SM Higgs production cross section are derived. This channel allows to exclude at $95 \% \mathrm{CL}$ a SM Higgs boson within a mass range of $300 \mathrm{GeV} \leq m_{H} \leq 322 \mathrm{GeV}$ or $353 \mathrm{GeV} \leq m_{H} \leq 410 \mathrm{GeV}$.

\section{$2.6 \mathrm{H} \rightarrow \mathrm{ZZ} \rightarrow 2 l 2 v$ searches}

A search for the SM Higgs boson in the mass range between 200 and $600 \mathrm{GeV}$ has been performed in the $\mathrm{H} \rightarrow \mathrm{ZZ} \rightarrow 2 l 2 v$ (where $l$ represents electrons or a muons) channel using $4.7 \mathrm{fb}^{-1}$ of proton proton collision data recorded at a centre-of-mass energy of $7 \mathrm{TeV}$ [26]. This channel is characterized by a final state with a large missing energy coming from the two neutrinos. The missing transverse momentum resolution is affected by the level of pile-up, resulting in a significant change in the signal to background ratio between low and high pile-up data taking periods. To retain the best sensitivity, the search is therefore split between the earlier "low pile-up data" $\left(2.3 \mathrm{fb}^{-1}\right)$ and the later "high pile-up data" $\left(2.4 \mathrm{fb}^{-1}\right)$ periods. In order to further increase the sensitivity, the search is also subdivided into a low Higgs boson mass region $\left(m_{H}<280 \mathrm{GeV}\right)$ and a high one $\left(m_{H} \geq 280 \mathrm{GeV}\right)$ where dedicated cuts are applied to two important discriminating variables used to reduce the background contamination: the transverse missing energy $\left(\mathbf{E}_{\mathrm{T}}^{\text {miss }}\right)$ and the azimuthal angle between the two leptons $\left(\Delta \phi_{l l}\right)$. These cuts reduce significantly the backgrounds from processes with no or modest genuine missing transverse momentum originating from unobserved neutrinos. Backgrounds modelling is validated and normalized from data. The main systematic uncertainties belong to the same categories already 
presented in the previous sections.

Finally the discriminating variable used in the final likelihood fit to data is the transverse mass $m_{\mathrm{T}}$, defined as $m_{\mathrm{T}}^{2}=\left.\left[\sqrt{m_{Z}^{2}+\left|\mathbf{p}_{\mathrm{T}}^{l l}\right|^{2}}+\sqrt{m_{Z}^{2}+\mid \mathbf{p}_{\mathrm{T}}^{\text {miss }}}\right]\right|^{2}-\left[\mathbf{p}_{\mathrm{T}}^{l l}+\mathbf{p}_{\mathrm{T}}^{\text {miss }}\right]^{2}$. Upper limits on the Higgs boson production cross section are derived and the production of a SM Higgs boson with a mass in the range $319 \mathrm{GeV} \leq m_{H} \leq 558 \mathrm{GeV}$ is excluded at the $95 \% \mathrm{CL}$.

\section{Conclusions}

Searches for an heavy neutral Higgs-like particle in the context of Standard Model and beyond have been presented. Final states of the WW and ZZ decay channels, sensitive to the high mass range, have been exploited up to $1 \mathrm{TeV}$ and exclusion limits on the SM Higgs boson mass as well as upper limits on production cross section times branching ratio have been set.

\section{References}

[1] ATLAS Collaboration, Phys. Lett. B 716, 1 (2012), 1207.7214

[2] CMS Collaboration, Phys. Lett. B 716, 30 (2012), 1207. 7235

[3] ATLAS Collaboration (2013), 1307.1427

[4] ATLAS Collaboration (2013), 1307.1432

[5] CMS Collaboration (2013), CMS-PAS-HIG-13-005

[6] A. Hill, J. van der Bij, Phys.Rev. D36, 3463 (1987)

[7] M. Veltman, F. Yndurain, Nucl.Phys. B325, 1 (1989)

[8] T. Binoth, J. van der Bij, Z.Phys. C75, 17 (1997), hep-ph/9608245

[9] R. Schabinger, J.D. Wells, Phys. Rev. D. 72, 093007 (2005), hep-ph/0509209

[10] B. Patt, F. Wilczek (2006), hep-ph/0605188

[11] LHC Higgs Cross Section Working Group, S. Heinemeyer, C. Mariotti, G. Passarino, R. Tanaka (Eds.) (2013), 1307.1347

[12] ATLAS Collaboration, ATLAS-CONF-2013-034 (2013)

[13] T.D. Lee, Phys. Rev. D. 8, 1226 (1973)

[14] J.F. Gunion, H.E. Haber, Phys. Rev. D. 67, 075019 (2003), hep-ph/0207010

[15] G. Branco, P. Ferreira, L. Lavoura, M. Rebelo, M. Sher et al., Phys. Rept. 516, 1 (2012), 1106.0034

[16] S. Goria, G. Passarino, D. Rosco, Nucl.Phys. B864, 530 (2012), 1112 . 5517

[17] J.M. Campbell, R.K. Ellis, C. Williams, JHEP 1110, 005 (2011), 1107.5569

[18] N. Kauer (2012), 1201. 1667

[19] G. Passarino, JHEP 1208, 146 (2012), 1206. 3824

[20] M. Bonvini, F. Caola, S. Forte, K. Melnikov, G. Ridolfi (2013), 1304 . 3053

[21] ATLAS Collaboration, ATLAS-CONF-2013-067 (2013)

[22] ATLAS Collaboration, ATLAS-CONF-2013-027 (2013)

[23] ATLAS Collaboration, ATLAS-CONF-2013-013 (2013)

[24] ATLAS Collaboration, Physics Letters B 718, 391 (2012)

[25] ATLAS Collaboration, Physics Letters B 717, 70 (2012)

[26] ATLAS Collaboration, Physics Letters B 717, 29 (2012) 